\title{
Electrochemiluminescent Detection of hNQO1 and Associated Drug Screening Enabled by Futile Redox Cycle Reaction
}

Yongjun Zheng, Zhenqiang Ning, Deng Pan, Dan Han, Yuan Xu, Songqin Liu, Yanfei Shen and Yuanjian Zhang*

Medical School, School of Chemistry and Chemical Engineering

Southeast University, Nanjing 211189 (China)

E-mail:Yuanjian.Zhang@seu.edu.cn

Abstract: Human $\mathrm{NAD}(\mathrm{P}) \mathrm{H}$ : quinone oxidoreductase 1 ( $\mathrm{hNQO1}$ ), a proteinase that engages in detoxification of quinones and capable of activating anti-tumor drugs, has drawn increasing attention as tumor biomarker and drug target. To date, the detection of hNQO1 primarily uses stimulus-responsive probes, involving metabolization of synthetic quinone-functionalized substrates, which however, remain challenging to improve the sensing signal-to-noise ratio, and are lack of sufficient stability. Herein, we report a facile but general way for hNQO1 detection and associated drug screening as well by ECL sensing of the metabolic $\mathrm{H}_{2} \mathrm{O}_{2}$ enabled by futile redox cycle reaction. Taking advantage of the intrinsic circulatory amplification and the luminol-modified nickel foam electrode, the sensing system exhibited a record-level performance in electrochemiluminescent detection of hNQO1. The same strategy was also successfully applied to rapidly screening hNQO1-directed anti-tumor candidate drugs. The proposed new principle for $\mathrm{hNQO1}$ detection would stimulate $\mathrm{ECL}$ as a promising tool that combines diagnostic and drug screening functions for the popularization of proteinases in cancer management.

Keywords: hNQO1, Hydrogen peroxide, Electrochemiluminescence, Cancer diagnosis, Drug screening 
Development of highly sensitive and selective early diagnosis and preclinical drug screening tools are significant for the treatment of tumors but remains great challenges. ${ }^{[1]}$ Recently, many proteinases are discovered to be overexpressed in a variety of tumor tissues, offering prospective opportunities. ${ }^{[2]}$ Among them, Human $\mathrm{NAD}(\mathrm{P}) \mathrm{H}$ : quinone oxidoreductase 1 (hNQO1), a two-electron reductase responsible for detoxification of quinones, has drawn increasing attention not only in the tumor diagnosis but also as antitumor drug target owning to unique bioactivation ability. ${ }^{[3]}$ To date, several methods have been developed to detect hNQO1, mainly using stimulusresponsive probes, which involve the reduction of a synthetic benzoquinonefunctionalized compound into phenol by hNQO1, followed by the release of signal reporters (mostly of fluorophores) via self-immolation reactions (Figure 1a). ${ }^{[4]}$ Despite the great progress achieved, however, the stimulus-responsive routes to improve sensing signal-to-noise (SNR) or positive-to-negative ratio (PNR) are limited and highly sophisticated. ${ }^{[5]}$ Moreover, most of the benzoquinone-based probes are lack of sufficient stability, in particular under light-excitation, due to the tendency of photobleaching and hydrolysis. ${ }^{[6]}$ Therefore, exploring a concept-new method for rapid, selective, and sensitive detection of $\mathrm{hNQO} 1$ is greatly envisioned.

Hydrogen peroxide $\left(\mathrm{H}_{2} \mathrm{O}_{2}\right)$, a normal aerobic metabolite occurring in cells, engages as a central hub in many signaling routes in vivo, ranging from proliferation to aging. ${ }^{[7]}$ Interestingly, it is noted that during metabolization of some quinone-substrates by $\mathrm{hNQO} 1$ with the participation of molecular oxygen, $\mathrm{H}_{2} \mathrm{O}_{2}$ is also produced. ${ }^{[8]}$ From this point of view, the activity of hNQO1 can be alternatively determined by measuring the metabolically generated $\mathrm{H}_{2} \mathrm{O}_{2}$. As known, electrochemiluminescence (ECL) is a simple, fast and sensitive analysis method, ${ }^{[9]}$ including in detection of $\mathrm{H}_{2} \mathrm{O}_{2},{ }^{[10]}$ which well integrates the advantage of both electrochemistry and chemiluminescence. ${ }^{[11]}$ Moreover, ECL without excitation light shows near-zero background, a unique superiority over other light emission methods, such as fluorescence. ${ }^{[12]}$ However, to the best of our knowledge, the study of detecting $\mathrm{hNQO} 1$ via $\mathrm{H}_{2} \mathrm{O}_{2}$ product and the use of the ECL technique have been rarely explored so far.

Herein, we report a facile but general approach for biosensing hNQO1 through detecting metabolically generated $\mathrm{H}_{2} \mathrm{O}_{2}$ by using a natural substrate and a simple ECL electrode made of luminol-modified nickel foam (Figure 1b). 
Interestingly, the showcase metabolization of $\beta$-lapachone by hNQO1 drove a futile redox cycle, leading to an interesting intrinsic circulatory production of $\mathrm{H}_{2} \mathrm{O}_{2}$. Further aid by porous nickel foam electrode with a high loading of luminol, the detection system exhibited excellent sensitivity and selectivity for hNQO1 with record-level detection limit $(10 \mathrm{ng} / \mathrm{mL})$ and SNR ( 460). Moreover, the similar strategy was successfully extended to rapidly screen a variety of hNQO1-directed anti-tumor drugs. The proposed new principle of hNQO1 activity evaluation would greatly pave the popularization of hNQO1 in cancer diagnosis and the associated drug screening, in particular by ECL.

(a) Previous work

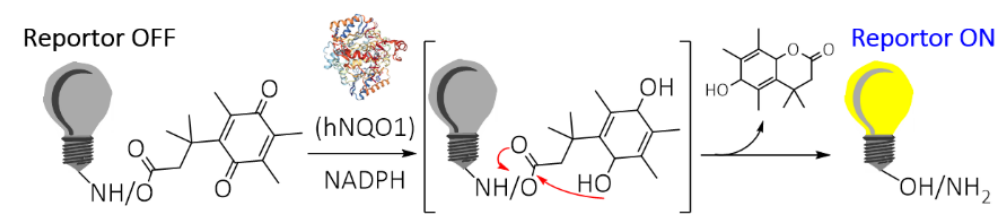

(b) This work

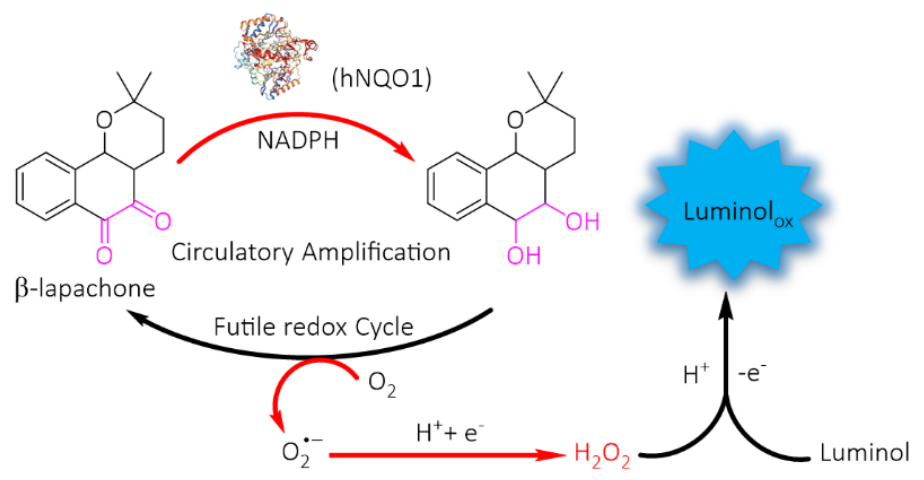

Figure 1. hNQO1 detection mechanism based on metabolization of (a) synthetic benzoquinone-based substrates that release a turned-on fluorophore and (b) natural $\beta$-lapachone substrate that in-situ generates $\mathrm{H}_{2} \mathrm{O}_{2}$, followed by triggering an ECL of luminol with an intrinsic circulatory amplification.

To meet the new detection principle, $\beta$-lapachone, which is isolated from Tabebuia avellanedae and has a high affinity to hNQO1, was used as a showcase natural substrate. During the metabolization of $\beta$-lapachone by hNQO1, $\mathrm{O}_{2}-{ }^{-*}$ would be produced with the participation of oxygen, and then turn into long-lived $\mathrm{H}_{2} \mathrm{O}_{2}$ (Figure 1b). After that, the accumulated $\mathrm{H}_{2} \mathrm{O}_{2}$ worked as the co-reagent to trigger the ECL of luminol. In this context, the concentration 
of hNQO1 could be indirectly reported by the ECL intensity of luminol. Moreover, it should be noted that by the participation of oxygen, a futile cycle would occur, making the unstable reduced substrate be reversibly recovered into the original state. Under such circulatory reactions, the concentration of the in-situ generated $\mathrm{H}_{2} \mathrm{O}_{2}$ and the subsequent $\mathrm{ECL}$ intensity would be intrinsically boosted, greatly favor of improving the sensitivity of hNQO1detection.
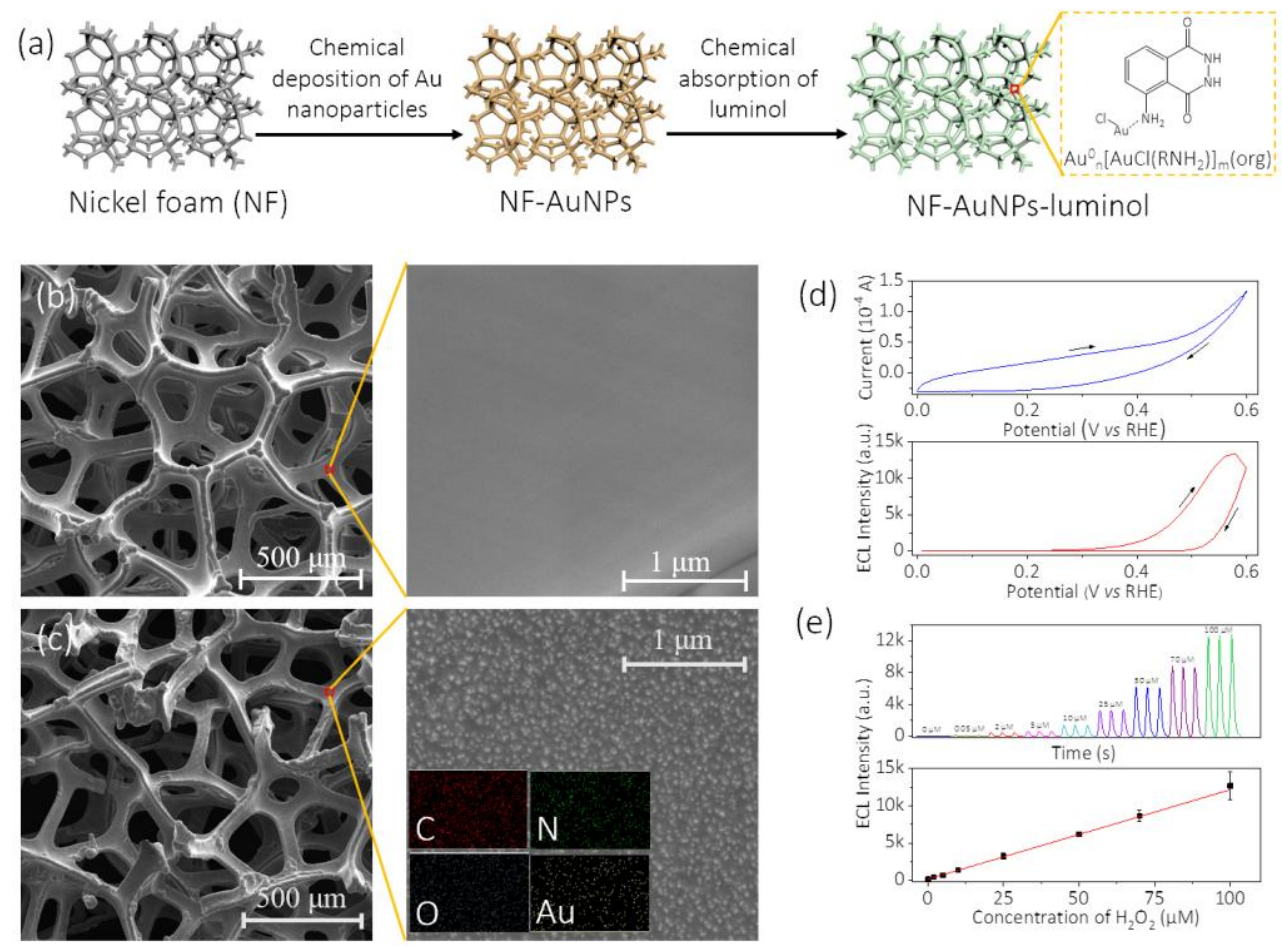

Figure 2. (a) Scheme of nickel foam (NF) working electrode and the loading of luminol via chemical deposited Au nanoparticles as linkers (NF/AuNPs/ luminol). SEM images of (b) bare NF and (c) NF/AuNPs/luminol. Inset: C, N, O, and Au EDS element mapping. (d) CV (top panel) and ECL (bottom panel) curves of NF/AuNPs/luminol in the presence of $\mathrm{H}_{2} \mathrm{O}_{2}(100 \mu \mathrm{M})$. (e) Plot of ECL intensity under different concentrations of $\mathrm{H}_{2} \mathrm{O}_{2}$ (top panel) and the liner fitting (bottom panel).

To improve the loading of luminol, a porous nickel foam was used as the working electrode. Gold nanoparticles (AuNPs) were chemically deposited in situ by reduction of $\mathrm{HAuCl}_{4}$ using $\mathrm{NaBH}_{4}$ in an aqueous solution, and then luminol was chemically absorbed on the AuNPs via the formation of Au-N bond. ${ }^{[13]}$ The concentration of $\mathrm{HAuCl}_{4}$ was optimized as $0.25 \mathrm{mM}$ to achieve a 
maximized ECL intensity (Figure $\mathrm{S} 1$ ). As shown in Figure $2 \mathrm{~b}$ and $2 \mathrm{c}$, the macroscopic 3D framework of the nickel foam was not altered after the deposition of AuNPs, but the smooth surface of the bare NF framework was observed in a higher magnification to be decorated with nanoparticles of average sizes ca. $20 \mathrm{~nm}$ (Figure S2). The inset EDS elemental mapping of the $\mathrm{NF} /$ AuNPs/luminol electrode and the detailed EDS spectrum (Figure S3) verified the co-existence of $\mathrm{Au}, \mathrm{C}, \mathrm{N}$, and $\mathrm{O}$. The stepwise fabrication of the electrode was also characterized by the electrochemical impedance spectroscopy (EIS, Figure S4). All these results confirmed the successful loading of luminol on NF via the AuNPs linkage.

In the next set of experiments, the performance of the as-prepared ECL electrode in detecting of standard $\mathrm{H}_{2} \mathrm{O}_{2}$ aqueous solution was evaluated. As shown in Figure 2d, the ECL intensity at NF/AuNPs/luminol electrode increased from $0.3 \mathrm{~V}$ and achieved its maximum at $0.58 \mathrm{~V}$; meanwhile, the anodic current, contributed by the oxidation of $\mathrm{H}_{2} \mathrm{O}_{2}$ and luminol, gradually improved. This phenomenon consisted of the ECL behavior of luminol in previous reports (Eq. $1-4)$.

Luminol $\stackrel{e^{-}, 2 H^{+}}{\longrightarrow}$ Luminol $^{-}$

$\mathrm{H}_{2} \mathrm{O}_{2} \stackrel{e^{-}}{\longrightarrow} \mathrm{O}_{2}{ }^{-}+2 \mathrm{H}^{+}$

Luminol $^{-}+\mathrm{O}_{2}{ }^{-} \longrightarrow$ Luminol $_{\mathrm{ox}}{ }^{*}+\mathrm{N}_{2}$

Luminol $_{\text {ox }}{ }^{*} \longrightarrow$ Luminol $_{\text {ox }}+h v$

Moreover, the NF/AuNPs/luminol electrode exhibited excellent stability by consecutive cyclic potential scans with a relative standard deviation (RSD) of $1.62 \%$ (Figure S5). Accordingly, with the increase of the concentration of $\mathrm{H}_{2} \mathrm{O}_{2}$ $(0-100 \mu \mathrm{M})$, the ECL intensity successively enhanced in a wide linear range between 0.05 and $100 \mu \mathrm{M}$ with a detection limit of $47 \mathrm{nM}$ (Figure 2e). As a control, a luminol modified glassy carbon electrode using AuNPs as linkers (GCE/AuNPs/luminol) was also constructed (Figure S6, S7). In contrast, under the same conditions, the detection limit at GCE/AuNPs/luminol electrode was $980 \mathrm{nM}$ (Figure S8), more than 20 times poorer than that at NF/AuNPs/luminol electrode, mainly owing to the porous structure of NF that could load more luminol than the most-commonly used GCE; nonetheless, NF is even much cheaper than GCE. The excellent sensing performance of the 
$\mathrm{NF} / \mathrm{AuNPs} /$ luminol electrode for $\mathrm{H}_{2} \mathrm{O}_{2}$ was supposed to lay a foundation for further highly sensitive detection of $h N Q O 1$ that in-situ generated $\mathrm{H}_{2} \mathrm{O}_{2}$ during metabolization of substances.
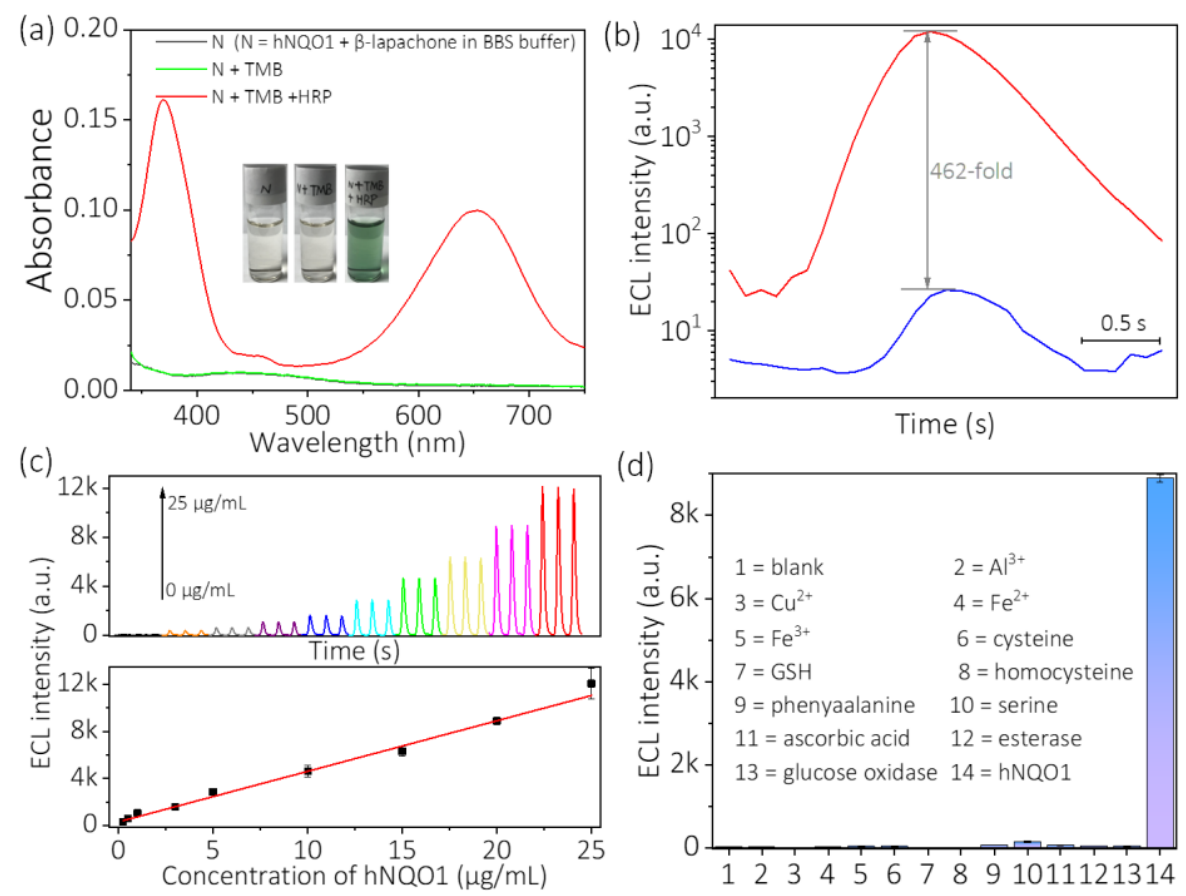

Figure 3. (a) UV-vis absorption spectra of the mixture of $\beta$-lapachone and hNQO1 in BBS buffer, and that with successive addition of TMB and HRP. (b) $\mathrm{ECL}$ intensity of NF/AuNPs/luminol in the BBS buffer with or without hNQO1 (25 $\mu \mathrm{g} / \mathrm{mL})$. (c) Plot of ECL intensity under different concentrations of hNQO1 (top panel) and the liner fitting (bottom panel). (d) ECL intensity of $\mathrm{NF} /$ AuNPs/luminol in the presence of hNQO1 and the potential interferences: 1 (blank), metal ions (1-5, $0.1 \mathrm{mM})$, small molecules (6-10, $0.1 \mathrm{mM})$, enzymes $(12-13,50 \mu \mathrm{g} / \mathrm{mL})$ and $14(\mathrm{hNQO1}, 20 \mu \mathrm{g} / \mathrm{mL})$.

Before applying NF/AuNPs/luminol in sensing of hNQO1, the in-situ generation of $\mathrm{H}_{2} \mathrm{O}_{2}$ in the metabolization of substances by hNQO1 was confirmed beforehand using the classical 3,3',5,5'-tetramethylbenzidine (TMB) color-change reaction in the presence of horseradish peroxidase (HRP). ${ }^{[14]}$ As shown in Figure 3a, when TMB and HRP were simultaneously added to the borate buffer solution (BBS) containing the mixture of $\beta$-lapachone and $\mathrm{hNQO1}$, the UV-vis absorption spectrum and color of the solution altered significantly, 
while that of the controls not, evidently indicating $\mathrm{H}_{2} \mathrm{O}_{2}$ was in-situ generated during the metabolization of substances by hNQO1.

After further optimization of the reaction time for hNQO1-driven metabolization (Figure S9), the ECL response of the NF/AuNPs/luminol to different concentrations of $\mathrm{hNQO} 1$ was monitored. As shown in Figure $3 \mathrm{~b}$ and $3 \mathrm{c}$, with the increase of the hNQO1 concentration $(0-25 \mu \mathrm{g} / \mathrm{mL})$, the $\mathrm{ECL}$ intensity was gradually enhanced with a linear range between $0.25 \mu \mathrm{g} / \mathrm{mL}-25$ $\mu \mathrm{g} / \mathrm{mL}\left(R^{2}=0.995\right)$ and a detection limit of $10 \mathrm{ng} / \mathrm{mL}$. Notably, such outstanding performance was already superior to most of the previous methods in sensing of hNQO1 (Figure S10 and Table S1). It was supposed that both the intrinsic circulatory amplification enabled by the futile redox cycle in the metabolization and the innovative designed porous NF/AuNPs/luminol ECL working electrode played crucial roles.

In order to evaluate whether the sensing system can be applied to complex biological systems, the selectivity and reliability tests were carried out. Various potential interferences in the organism, including metal ions $\left(\mathrm{Al}^{3+}, \mathrm{Cu}^{2+}, \mathrm{Fe}^{2+}\right.$, and $\mathrm{Fe}^{3+}$ ), small molecules (cysteine, GSH, homocysteine, phenylalanine, serine, and ascorbic acid) and some enzymes (esterase and glucose oxidase) were measured. As shown in Figure 3d, only hNQO1 could give rise to a significant ECL intensity, clearly showing that the sensing system had an excellent selectivity for hNQO1 and was supposed to well avoid false-positive measurements. In addition, the detection of hNQO1 in serum was also evaluated. As shown in Table S2, five spiked human serum samples containing $20 \mu \mathrm{g} / \mathrm{mL}, 10 \mu \mathrm{g} / \mathrm{mL}, 5 \mu \mathrm{g} / \mathrm{mL}, 1 \mu \mathrm{g} / \mathrm{mL}$ and $0.5 \mu \mathrm{g} / \mathrm{mL}$ of hNQO1 were tested, indicative of a good detection performance between $0.5-20 \mu \mathrm{g} / \mathrm{mL}$ with the recovery rate between $94.62 \%$ and $101.36 \%$, which well met the actual testing needs. 

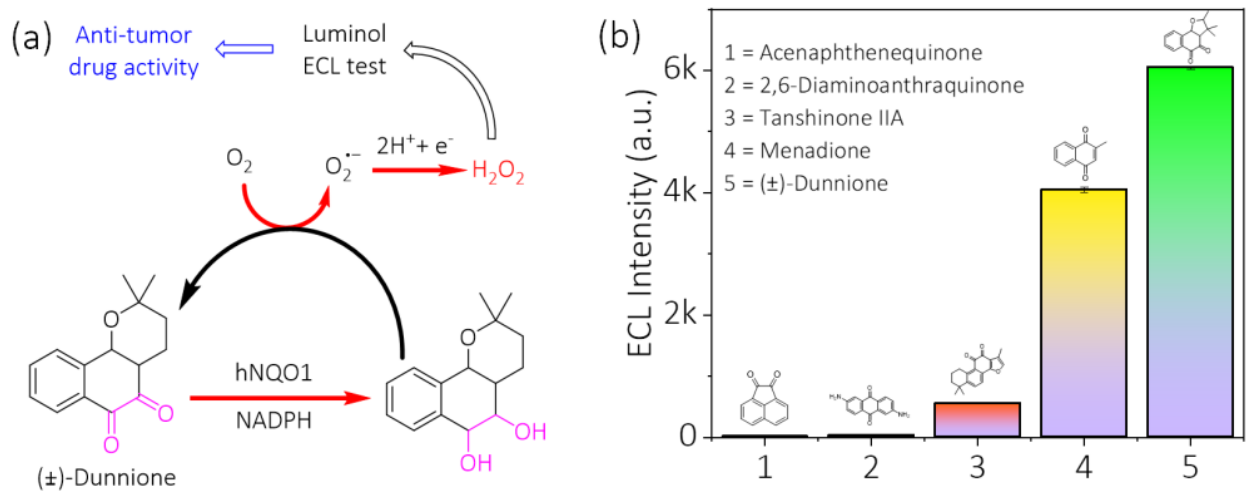

Figure 4. (a) Mechanism of anti-tumor activity analysis with ( \pm )-Dunnione as the model drug in the presence of $\mathrm{hNQO} 1$ via measuring $\mathrm{H} 2 \mathrm{O} 2$-product that triggered ECL. (b) ECL intensity at NF/AuNPs/luminol in the presence of different anti-tumor drug candidates $(10 \mu \mathrm{M})$.

In addition to detecting hNQO1, the potential of the same sensing principle was also investigated for the application in the screening of relevant anti-tumor drugs. As known, $\mathrm{H}_{2} \mathrm{O}_{2}$ is an important active species produced in the metabolization of $\mathrm{hNQO1-directed} \mathrm{anti-cancer} \mathrm{drugs.}{ }^{[8 \mathrm{~b}, 15]}$ In this context, the activity of the drugs could also be quickly evaluated by sensing of the in-situ generated $\mathrm{H}_{2} \mathrm{O}_{2}$ when the metabolization occurred. Thus, by fixing the concentration of hNQO1, the activity of different candidate drugs could be facilely ranked according to the ECL intensity (Figure $4 \mathrm{a}$ ), which would be very valuable in guiding clinic drug research. To realize this assumption, the performance of a variety of potential drugs was evaluated. As shown in Figure 4b, two ordinary quinone compounds (acenaphthenequinone and 2,6diaminoanthraquinone) showed no evident ECL. In contrast, another three compounds (tanshinone IIA, menadione and ( \pm )-Dunnione) that have been reported to be as the efficient anti-tumor candidate drugs for hNQO1, exhibited profound $\mathrm{ECL}$ with a successive increase in intensity, consistent with previously reported results obtained from cell research. ${ }^{[16]}$ It should be noted that compared to traditional cell screening method that generally takes several hours or an even longer time, the coupling of metabolization by hNQO1 and the ECL measurement only need tens of minutes. Therefore, the utilization of metabolically generated $\mathrm{H}_{2} \mathrm{O}_{2}$-product by $\mathrm{hNQO} 1$ and the $\mathrm{ECL}$ technique held 
great potential in high-throughput anti-tumor drug screening, especially for preclinical drug studies.

In summary, we report a new principle to detect hNQO1 through the sensing of $\mathrm{H}_{2} \mathrm{O}_{2}$ generated by futile redox cyclic metabolization of a natural substrate, which further triggered an ECL signal readout of luminol. Thanks to the intrinsic circulatory amplification and the innovative designed porous NF/AuNPs/luminol working electrode, this ECL sensing system exhibited a record-level performance in the detection of hNQO1 with a detection limit of (10 $\mathrm{ng} / \mathrm{mL}$ ) and SNR of $\sim 460$, excellent selectivity and reliability. Moreover, the similar format was further successful extended to rapidly ranking the activity of a variety of hNQO1-directed anti-tumor candidate drugs. This work would not only open a new way to facilely detect $\mathrm{hNQO1}$, but also stimulate ECL as a promising tool that combines diagnostic and drug screening functions for proteinases in cancer management.

\section{Acknowledgements}

This work was supported by the National Natural Science Foundation of China (21775018, 21675022), the Natural Science Foundation of Jiangsu Province (BK20160028), the Open Funds of the State Key Laboratory of Electroanalytical Chemistry (SKLEAC201909), and the Fundamental Research Funds for the Central Universities.

[1] a) R. Etzioni, N. Urban, S. Ramsey, M. McIntosh, S. Schwartz, B. Reid, J. Radich, G. Anderson, L. Hartwell, Nat. Rev. Cancer 2003, 3, 243.; b) E. J. Kwon, J. S. Dudani, S. N. Bhatia, Nat. Biomed. Eng. 2017, 1, 1.; c) F. Eduati, R. Utharala, D. Madhavan, U. P. Neumann, T. Longerich, T. Cramer, J. Saez-Rodriguez, C. A. Merten, Nat. Commun. 2018, 9, 2434; d) Y. Wu, R. D. Tilley, J. J. Gooding, J. Am. Chem. Soc. 2018, 141, 1162.; e) S. Lee, E. J. Cha, K. Park, S. Y. Lee, J. K. Hong, I. C. Sun, S. Y. Kim, K. Choi, I. C. Kwon, K. Kim, C. H. Ahn, Angew. Chem. Int. Ed. 2008, 47, 2804; Angew. Chem. 2008, 120, 2846.

[2] a) H. Singh, K. Tiwari, R. Tiwari, S. K. Pramanik, A. Das, Chem. Rev. 2019, 119, 11718.; b) L. Wu, X. Qu, Chem. Soc. Rev. 2015, 44, 2963. 
[3] a) M. A. B. Rongbao Li, Paul Talay, L. Mario Amzel, Proc. Natl. Acad. Sci. USA 1995, 92, 8846.; b) E. T. Oh, J. W. Kim, J. M. Kim, S. J. Kim, J. S. Lee, S. S. Hong, J. Goodwin, R. J. Ruthenborg, M. G. Jung, H. J. Lee, C. H. Lee, E. S. Park, C. Kim, H. J. Park, Nat. Commun. 2016, 7, 1.; c) K. Zhang, D. Chen, K. Ma, X. Wu, H. Hao, S. Jiang, J. Med. Chem. 2018, 61, 6983.; d) E. I. Parkinson, P. J. Hergenrother, Acc. Chem. Res. 2015, 48, 2715.; e) X. Li, Z. Liu, A. Zhang, C. Han, A. Shen, L. Jiang, D. A. Boothman, J. Qiao, Y. Wang, X. Huang, Y.-X. Fu, Nat. Commun. 2019, $10,1$.

[4] a) S. U. Hettiarachchi, B. Prasai, R. L. McCarley, J. Am. Chem. Soc. 2014, 136, 7575.; b) W. C. Silvers, B. Prasai, D. H. Burk, M. L. Brown, R. L. McCarley, J. Am. Chem. Soc. 2013, 135, 309.; c) S. Son, M. Won, O. Green, N. Hananya, A. Sharma, Y. Jeon, J. H. Kwak, J. L. Sessler, D. Shabat, J. S. Kim, Angew. Chem. Int. Ed. 2019, 58, 1793.; Angew. Chem. 2019, 131, 1.; d) C. Zhang, Q. Z. Zhang, K. Zhang, L. Y. Li, M. D. Pluth, L. Yi, Z. Xi, Chem. Sci. 2019, 10, 1945.

[5] a) O. Green, S. Gnaim, R. Blau, A. Eldar-Boock, R. Satchi-Fainaro, D. Shabat, J. Am. Chem. Soc. 2017, 139, 13243.; b) F. Liu, X. Shi, X. Liu, F. Wang, H.-B. Yi, J.-H. Jiang, Chem. Sci. 2019, 10, 9257.

[6] a) G. Hong, A. L. Antaris, H. Dai, Nat. Biomed. Eng. 2017, 1, 1.; b) H. Tong, Y. Zheng, L. Zhou, X. Li, R. Qian, R. Wang, J. Zhao, K. Lou, W. Wang, Anal. Chem. 2016, 88, 10816.

[7] a) Z.-X. Y. Maitrayee Sundaresan, Victor J. Ferrans, Kai kobad Irani, Toren Finkel, Science 1995, 270, 296; b) H. Sies, J. Biol. Chem. 2014, 289, 8735.

[8] a) X. Huang, Y. Dong, E. A. Bey, J. A. Kilgore, J. S. Bair, L. S. Li, M. Patel, E. I. Parkinson, Y. Wang, N. S. Williams, J. Gao, P. J. Hergenrother, D. A. Boothman, Cancer Res. 2012, 72, 3038.; b) X. Huang, E. A. Motea, Z. R. Moore, J. Yao, Y. Dong, G. Chakrabarti, J. A. Kilgore, M. A. Silvers, P. L. Patidar, A. Cholka, F. Fattah, Y. Cha, G. G. Anderson, R. Kusko, M. Peyton, J. Yan, X. J. Xie, V. Sarode, N. S. Williams, J. D. Minna, M. Beg, D. E. Gerber, E. A. Bey, D. A. Boothman, Cancer Cell 2016, 30, 940.

[9] a) M. M. Richter, Chem. Rev. 2004, 104, 3003.; b) Z. Liu, W. Qi, G. Xu, Chem. Soc. Rev. 2015, 44, 3117. 
[10]a) S. A. Kitte, W. Gao, Y. T. Zholudov, L. Qi, A. Nsabimana, Z. Liu, G. Xu, Anal. Chem. 2017, 89, 9864.; b) H. Cui, W. Wang, C. F. Duan, Y. P. Dong, J. Z. Guo, Chemistry 2007, 13, 6975.; c) X. Jiang, H. Wang, R. Yuan, Y. Chai, Anal. Chem. 2018, 90, 8462.

[11]H. Qi, C. Zhang, Anal. Chem. 2020, 92, 524.

[12]L. Li, Y. Chen, J. J. Zhu, Anal. Chem. 2017, 89, 358.

[13] a) M. Sethi, M. R. Knecht, Langmuir 2010, 26, 9860.; b) S. M. Ashavani Kumar, P.R. Selvakannan, Renu Pasricha,, a. M. S. A. B. Mandale, Langmuir 2003, 19, 6277.

[14]a) Y. Nosaka, A. Y. Nosaka, Chem. Rev. 2017, 117, 11302.; b) P. Zhang, D. Sun, A. Cho, S. Weon, S. Lee, J. Lee, J. W. Han, D. P. Kim, W. Choi, Nat. Commun. 2019, 10, 940.

[15]a) M. S. Beg, X. Huang, M. A. Silvers, D. E. Gerber, J. Bolluyt, V. Sarode, F. Fattah, R. J. Deberardinis, M. E. Merritt, X. J. Xie, R. Leff, D. Laheru, D. A. Boothman, J. Surg. Oncol. 2017, 116, 83.; b) E. A. B. Kathryn E. Reinicke, Melissa S. Bentle, John J. Pink, Stephen T. Ingalls, Charles L. Hoppel, Rosana I. Misico, Gisela M. Arzac, Gerardo Burton, William G. Bornmann, Damon Sutton, Jinming Gao and David A. Boothman, Clin. Cancer Res. 2005, 11, 3055.; c) E. A. Bey, K. E. Reinicke, M. C. Srougi, M. Varnes, V. E. Anderson, J. J. Pink, L. S. Li, M. Patel, L. Cao, Z. Moore, A. Rommel, M. Boatman, C. Lewis, D. M. Euhus, W. G. Bornmann, D. J. Buchsbaum, D. R. Spitz, J. Gao, D. A. Boothman, Mol. Cancer Ther. 2013, 12, 2110.

[16]a) J. Bian, B. Deng, L. Xu, X. Xu, N. Wang, T. Hu, Z. Yao, J. Du, L. Yang, Y. Lei, X. Li, H. Sun, X. Zhang, Q. You, Eur. J. Med. Chem. 2014, 82, 56.; b) J. Bian, L. Xu, B. Deng, X. Qian, J. Fan, X. Yang, F. Liu, X. Xu, X. Guo, X. Li, H. Sun, Q. You, X. Zhang, Bioorg. Med. Chem. Let. 2015, 25, 1244.; c) J. Bian, X. Li, N. Wang, X. Wu, Q. You, X. Zhang, Eur. J. Med .Chem. 2017, 129, 27. 